\title{
The Flaw of the Traditional Plastic Potential Theory and the Correction in the Geotechnical Application
}

\author{
Siyi DU \\ School of Civil Engineering \\ Zhengzhou University \\ Zhengzhou, China \\ e-mail: dsy3227@163.com \\ Penghui NIE \\ School of Civil Engineering \\ Zhengzhou University \\ Zhengzhou, China
}

\author{
Zhiling WANG \\ School of Resources and Environment \\ North China University of Water Resources and Electric \\ Power \\ Zhengzhou, China
}

\author{
Hailin LIU \\ School of Civil Engineering \\ Zhengzhou University \\ Zhengzhou, China
}

\begin{abstract}
Given the basis that there is no perfect theoretical of the simple and practical geotechnical elastoplastic model which has been set up, triaxial compression test results cannot provide elastic-plastic analytical formula. This article embarks from the basic definition, according to the variation characteristics of micro material under stress, analyses the main reason for traditional plastic potential theory which is not applicable to the geotechnical. Without considering yield stress increasing produce the plastic volumetric strain, plastic work is generally regarded as the plastic potential energy; Postulate of Ilyushin is contrary to the basic hypothesis and ignores the function of the definition of the basic features. Correction method is put forward according to the principle of conservation of energy to define the potential energy of soil plasticity as plastic volume of work done by external force, with the truth of octahedral axis compression plastic potential function instead of Mises's assumption; Natural shear shrinkage, Drucker's inference postulate, dilatancy, by decomposing the plastic volume dilatancy change we get the shear shrinkage, the plastic potential function attached plastic volume expansion line. This kind of research method change Cambridge model to analyze various soil type under axial compression, theoretical derivation and expression is more simple; In reshaping of the shear shrinkage and dilatancy, saturated and unsaturated soil in the application, the results show that: through a correction coefficient of saturated soil and unsaturated soil deformation calculation value, which can effectively describe the triaxial test measurements; Because the triaxial test is widely used in deformation calculation, geotechnical plastic mechanics development towards the direction of the simple and practical is done to explore a new path.
\end{abstract}

Keywords-yield stress; triaxial test; energy equation; the plastic potential energy; the plastic potential function

\section{INTRODUCTION}

The geotechnical is natural elastic-plastic material and its pore structure is obvious, with plastic deformation significantly. Describing the stress-strain relationship should use the elastoplastic model. Early geotechnical elastoplastic model theory is based on the traditional plastic potential theory [1]. In the history of soil mechanics, the landmark Cambridge model is based on this and the triaxial test results of saturated soil. Its outstanding advantage is with less parameters and can be confirmed according to the conventional triaxial test. A large number of the test results show that suitable for metal of the traditional potential theory used in the geotechnical will be many unconformity to the actual situation, they are as below in summary [2]: (1) Be contrary to the associated flow rule and drucker postulate (2) The direction of plastic strain increment and stress state are not uniqueness (3) Unable to calculate the plastic strain produced by the rotation of the stress spindle (4) The single yield surface cannot describe the dilatancy and the shear shrinkage at the same time. In order to adapt to the deformation mechanism of rock and soil, various types of elastoplastic model are constantly emerging. Due to modeling method are basically the same: , on the premise of assumption (damage, the form of the yield function and hardening parameters, flow rule and hardening rule shall be presumed), consistent with a test or measured results as correct, their deducing are often complex, arbitrary and their parameters generally cannot all be determined by routine test. So they are not suitable for general engineering use. In engineering, soil stress state in general can be simplified as the axial compression, simulating the stress state in the routine is triaxial test. The article obtains from the basic definition, based on the simple principle of physics, mathematics and the axial compression of soil characteristics, analyze the reason of traditional potential theory for geotechnical appearing afore-mentioned four kinds of phenomena, use the real plastic potential function to replace the assumption of traditional plastic potential theories, established the elastic-plasticl analytic formula of soil under axial compression, qualitatively apply to various types of soil, correcting the quantitative problem based on experimental study. 


\section{The Flaw of the Traditional Plastic Potential THEORY AND THE REASON OF NOT APPLICABLE TO ROCK AND SOIL}

Based on the assumption that there is a kind of plastic potential function which is similar to elastic potential function in the state of plastic flow, its gradient direction determines the direction of the plastic flow. This is Mises (1928) proposed the traditional plastic potential theory, mathematical expressions for [3].

$$
d \varepsilon_{i j}^{p}=d \lambda \frac{\partial \boldsymbol{Q}}{\partial \sigma_{i j}}
$$

Where $\mathrm{d} \lambda$ : the negative factors

Drucker postulate exported the yield surface convex outside equal to the plastic potential surface by stress cycle the external force do nonnegative work, forming the associated flow rule that plastic strain increment direction will be orthogonal to yield surface, the postulate is only applicable to the strain hardening material. Drucker postulate exported the yield function equal to the plastic potential function by strain cycle the external force do nonnegative work, applying strain hardening and softening materials. Because it references the Drucker postulate, it requires meeting the associated flow rule. Drucker postulate and Ilyushin postulate is the basis of the traditional plastic mechanics, they put the plastic potential function and the yield function closely linked together. There are two obvious defects of Ilyushin postulate: (1) basic hypothesis contrary to the basic definition, plastic deformation is an kind of irreversible deformation, strain loop in plastic state is impossible (2) ignore the basic properties of the function, that a function has its domain, material hardening and softening stage does not belong to a domain. The nature is different, at the same time meet the associated flow rule determines that it shall not apply to the rock and soil. Ilyushin Postulate defect is the defect of the traditional potential theory. The traditional plastic potential theory has a hidden defect: expression is too general, change additional internal force to external force without taking micro unit, without analyzing the definition of the plastic potential energy, $Q\left(\sigma_{i j}\right)$ is a kind of assumption. Applying to metal, mainly metal only has plastic shear strain.

There are two kinds of geotechnical plastic strain (looking shear strain of soil as plastic), finding out the reason of yield stress increased before establishing a yield function is necessary, make the plastic potential energy conform to the actual definitions. The reason that the yield stress increases after material produce plastic deformation is the changes in the microstructure. In terms of energy analysis, the external force to work changes the micro unit plastic potential energy. Metal micro structure change is the line of compression (or line elongation), described by the plastic shear strain. Changes of soil microstructure is pore, soil particle not change (ignoring the influence of wear and breakage of its edges and corners), described in the plastic volumetric strain, rock and soil microstructure changes divided into the micro fracture and fracture pressure ${ }^{[4]}$, also described by the plastic volume change. Drucker postulate shall not apply to the rock and soil, mainly because of regarding the geotechnical plastic work as plastic potential energy causing yield development degree of hardening parameters measured in various assumptions [3]: (1) plastic work (2) the plastic volume strain (3) the plastic shear strain (4) plastic strain (5) some combination of the plastic volumetric strain and the plastic shear strain. Plastic strain is synthetic by two strains. Plastic work and both strain component has a variety of corresponding relation. Producing shear strain does not improve the yield stress. Reaching plastic work equal stress points produce different plastic volumetric strain and shear strain. Yield stress is different, so the plastic potential function is not equal to the yield function.

Geotechnical plastic potential definition can be described by plastic volume work. The hardening parameter is plastic volume strain. The assumption of Cambridge model conform the actual. Strain of volume strain and three dimension (plane strain state become $2 \mathrm{~d}$ cant) is linear. Taking octahedron as micro unit, formula (1) applies to metal and geotechnical. Plastic strain increment direction of octahedron is ruled by the proportion of shear strain increment and plastic volumetric strain increment. Looking from the perspective of the calculus, stress state decides the direction of plastic strain increment. Being described by a function need component stress and plastic strain component correspond one-to-one. Metal is Isotropic. Isotropic stress does not produce plastic strain. Plastic shear strain is controlled by the deviatoric stress. Without regulating the loading direction, stress components and plastic components is one to one relationship. There is cross effects of stress and strain of soil. Through consolidated undrained (CU) or consolidation drainage (U) test, stress path under pressure of axial is controlled by consolidation compression. Stress components and plastic strain components is one to one relationship. The previous model is applicable to arbitrary loading direction. Stress components and the direction of plastic strain increment are not unique. In fact, no matter whether regulate loading direction, loading direction of determining soil strength parameters is the model directly applicable loading direction.

Triaxial test without the spindle rotation problem, as other static load test does. Matsuoka calculation method is too complicated. Researching plastic strain produced by the rotation of the spindle should take dynamic triaxial test. Octahedron stress component belongs to stress invariants and the deformation can be considered in modified category.

Volume force and volume strain are reverse in direction. To reduce the plastic potential energy, we should change dilatancy to shear shrinkage before calculating the deformation; otherwise, it is not in conformity with the mechanics principle. The following example given is to illustrate the problem. 


\section{The Calculation Formula of Plastic Potential FUNCTION AND THE PLASTIC STRAIN OF OCTAHEDRON UNDER AXIAL COMPRESSION}

Soil produces shear strain. At the same time produce the plastic volumetric strain, before reaching the infinite plastic state. When loading direction is fixed, the proportion of them depends on the stress state, and it can be described in energy equation of differential form. Cutting out an octahedron from the normal consolidation soil, in axial compression, the energy equation for

$$
p^{\prime} d \varepsilon_{8}^{p}+\tau_{8} d\left(\frac{\gamma_{8}}{2}\right)=f_{8}\left(p^{\prime}+p_{m}\right) d\left(\frac{\gamma_{8}}{2}\right)
$$

Where, $\tau 8$ and $\mathrm{p}^{\prime}$ is the octahedral shear stress and normal stress effectively; $d \varepsilon_{8}^{p}$ and $\mathrm{d}\left(\frac{\gamma_{8}}{2}\right)$ :octahedron plastic strain and normal angle change represented by differential incremental; $\gamma_{8}$ : octahedral shear strain; $f_{8}$ : the ratio of the octahedral shear stress and effective stress in unlimited plastic state; pm: the internal stress caused by the suction, as to saturated soil and sandy soil, $\mathrm{pm}=0 . \mathrm{f8}$ is inherent in soil [5], called true friction parameter. The formula of effective stress of unsaturated soil $\mathrm{p}$ and internal stress $\mathrm{pm}$ refer to literature [6] or [7].

Change the stress and strain coordinate plane of fantasy

(1) from $\left(\tau 8, \mathrm{p}^{\prime}\right),\left(\gamma_{8}, \varepsilon_{8}^{\mathrm{p}}\right)$ to $\left(\mathrm{q}, \mathrm{p}^{\prime}\right),\left(\varepsilon, \varepsilon_{\mathrm{v}}^{\mathrm{p}}\right)$, get

$$
q=\left[M+\left(-\frac{d \varepsilon_{v}^{p}}{d \varepsilon}\right)\right] p^{\prime}+M p_{m}
$$

In type the $\mathrm{q}$ and $\varepsilon$ : Generalized shear stress and the generalized shear strain, the calculation formula refer to literature [2]; $\varepsilon_{\mathrm{v}}^{\mathrm{p}}\left(=3 \varepsilon_{8}^{\mathrm{p}}\right)$ : Plastic volumetric strain; M: true strength parameters, switch f8 to numerical plane of (q, p); $\left(-\frac{\mathrm{d} \varepsilon_{\mathrm{v}}^{\mathrm{p}}}{\mathrm{d} \varepsilon}\right)$ : plastic volume expansion rate.

In axial compression, $\mathrm{M}$ is fixed. When $\left(-\frac{\mathrm{d} \varepsilon_{\mathrm{v}}^{\mathrm{p}}}{\mathrm{d} \varepsilon}\right)$ adding to the extreme value of zero, soil damage. Then formula (3) becomes damage equation. $\mathrm{M}$ is determined by saturated soil, having nothing to do with the saturation state [5, 6]. Overlapping the coordinate plane $(\mathrm{q}, \mathrm{p})$ and $\left(\varepsilon, \varepsilon_{\mathrm{v}}^{\mathrm{p}}\right), \frac{\mathrm{dq}}{\mathrm{dp}}$ and $\frac{\mathrm{d} \varepsilon}{\mathrm{d} \varepsilon_{\mathrm{v}}^{\mathrm{p}}}$ are the direction of stress increment and plastic strain increment. Connecting the plastic potential energy equal stress points together, we get strips of lines called equal plastic potential line, also known as the yield locus. Their expression is $\mathrm{Q}(\mathrm{q}, \mathrm{p})=\mathrm{k}$ (constant). These parallel curves come from $\mathrm{p}$ axis, ending with damage line, smoothly and continuously. Because of $\mathrm{dp}$ and $\mathrm{d} \varepsilon_{v}^{p}, \mathrm{dq}$ and $\mathrm{d} \varepsilon$ is epsilon, equal plastic potential line gradient (external normal) is consistent with the direction of plastic strain increment. Curve is convex. Convex curve is located in the first quadrant. The tangent slope is negative and External normal is positive slope, the formula is established

$$
\frac{d q}{d p}=-\frac{d \varepsilon_{v}^{p}}{d \varepsilon}
$$

Put above formula plug formula (3), we get a differential equation. Solving the equation through regarding the starting point coordinates of the equal plastic potential line as the initial conditions; we get a plastic potential function. On same plastic potential line, a stress points correspond to a stress path. The actual stress state may not move along it.

Dense sand and over-consolidated soil is in elastic state at the beginning of shear with volume shrinkage. After yield the body begins inflate. Formula (2) is applicable $(\mathrm{pm}=0)$. Cohesion of over-consolidated soil is caused by unloading, then loaded into the yield condition. It fully play and energy equation refer to literature [5]. For simple, adding the influence of cohesion to M, Formula (3) $(\mathrm{pm}=0)$ also applies. True strength parameters of dense sand and overconsolidated soils $\mathrm{M}$ is determine by yield point $\left(\mathrm{d} \varepsilon_{\mathrm{v}}^{\mathrm{p}}=0\right)$ stress state. Substituting plastic volume expansion rate $\left(-\frac{d \varepsilon_{v}^{p}}{d \varepsilon}\right)_{f}$ of the peak point into (2), we get the damage equation. Ordering $\mathbf{M}^{*}=\mathbf{M}+\left(-\frac{\mathrm{d} \varepsilon_{\mathrm{v}}^{\mathrm{p}}}{\mathrm{d} \varepsilon}\right)_{\mathrm{f}}$, which $\mathrm{M} *$ is call strength parameters. When dilatancy, $\mathrm{M}<\mathrm{M}$ *; when Shear shrinkage, $M=M^{*}$.

In coordinate plane of $(q, p)$, plastic potential line of dense sand and over-consolidated soils begin from the yield line and stop to damage line, smoothly and continuously. Because the dp and (-d $\left.\varepsilon_{v}^{\mathrm{p}}\right)$ are in the same direction while $\mathrm{dq}$ and $\mathrm{d} \varepsilon$ are in the same direction, equal plastic potential line gradient and plastic strain increment reverse. Curve is outer concave, having no intersection point with the $\mathrm{p}$ axis, so it is meaningless to find its expression. Seeking useful plastic potential function need to enlarge the domain of function, making it include $\mathrm{p}$ axis. Therefore, increasing the isotropical pressure, making load starting point revert to normal consolidation state. In axis compression $\mathrm{p}$ does positive work, at the same time increase the $\mathrm{q}$ ensuring $\mathrm{q} / \mathrm{p}$ is constant. Shear strain is not affected. Increase to the pressure of axial compression of the plastic volume strain effect through additional compensation. Actual operation without transformation stress, only need to add the dilatancy consumption of energy to M., namely increases $M$ to $M *$ under the premise of not affect formula (3) establish.

Ordering $d \varepsilon_{v}^{p}=d \varepsilon_{v}^{p}+d \varepsilon_{v 2}^{p}$, Substitute it into (3), the formula of $\mathrm{Pm}=0$, divided into the following two equations, get

$$
\frac{\mathrm{d} \varepsilon_{\mathrm{v} 1}^{\mathrm{p}}}{\mathrm{d} \varepsilon}=\mathbf{M}^{*}-\frac{\mathrm{q}}{\mathbf{p}^{\prime}}
$$




$$
\frac{d \varepsilon_{v 2}^{p}}{d \varepsilon}=M-M^{*}
$$

Formula (5) is the energy equation of Shear shrinkage of soil. Substituting formula (4) in the equation to get a differential equation, using equal plastic potential line starting point: $\mathrm{q}=0, \mathrm{p}^{\prime}=\mathrm{p} 0$ as initial condition to solve this equation. $\mathrm{Q}\left(\mathrm{q}, \mathrm{p}^{\prime}\right)=\mathrm{k}$ can be expressed as follow

$$
\frac{\mathrm{q}}{\mathrm{Mp}}-\ln \mathrm{p}^{\prime}=\ln \mathrm{p}_{\mathrm{o}}^{\prime}
$$

The above formula is yield equation of cambridge model, its function is convert plastic volume strain of the normal consolidated soil produced by shearing after consolidation to isotropic pressure $\mathrm{p} 0$, in order to distinguish yield criterion, loading and unloading. The formula form of isotropic consolidation plastic volumetric strain of unsaturated soil is the same as saturated soil, Substitute the formula into the above formula, written in differential form: $d \varepsilon_{v 1}^{p}$. Substitute $\mathrm{d} \varepsilon_{\mathrm{v} 1}^{\mathrm{p}}$ into formula (5) for $d \varepsilon$.Formula (7) are additional volume expansion line whose form is the same as Light Of Tibet assumption [8]. Substitute $d \varepsilon$ into formula (6) for $\mathrm{d} \varepsilon_{\mathrm{v} 2}^{\mathrm{p}}$, merge $\mathrm{d} \varepsilon_{\mathrm{v} 1}^{\mathrm{p}}, \mathrm{d} \varepsilon_{\mathrm{v} 2}^{\mathrm{p}}$, get

$$
d \varepsilon_{v}^{p}=\frac{M-z}{M^{*}-z} \frac{(\lambda-\kappa)}{\left(1+e_{0}\right)}\left(\frac{1}{p^{\prime}} d p^{\prime}+\frac{1}{M^{*}} d z\right)
$$

Formula of $\mathrm{z}\left(=\frac{q}{p}\right)$ : stress level; $\mathrm{e} 0$ : initial void ratio; $\lambda$ and $\kappa$ : compressive index and inflate index.

$\mathrm{Pm}$ is greater, while $\lambda$ is smaller. The relationship between them refers to literature [7]. $\mathrm{K}$ has nothing to do with saturated state. Regard axial compression starting condition $\mathrm{z}=0, \mathrm{p}_{0}^{\prime}=\gamma_{8}+\mathrm{p}_{\mathrm{b}}, \varepsilon_{\mathrm{v}}^{\mathrm{p}}=0, \varepsilon=0$ as the initial condition, integrate $d \varepsilon \mathrm{d} \varepsilon_{\mathrm{v}}^{\mathrm{p}}$, get

$\varepsilon_{v}^{p}=\frac{\lambda^{*}-\kappa}{1+e_{0}}\left[\frac{M-z}{M^{*}-z} \ln \frac{p^{\prime}}{p_{0}^{\prime}}+\frac{M^{*}-M}{M^{*}} \ln \frac{M^{*}-z}{M^{*}}+\frac{z}{M^{*}}\right)$

$$
\varepsilon=\frac{\lambda^{*}-\kappa}{1+e_{0}}\left[\frac{\ln p^{\prime}-\ln p_{0}^{\prime}}{M^{*}-z}-\frac{1}{M^{*}} \ln \frac{M^{*}-z}{M^{*}}\right]
$$

When the above two formulas used in the shear shrinkage soil, $\mathrm{M}=\mathrm{M} *$; when used in unsaturated soil, the calculating formula of $\mathrm{p}^{\prime}, \lambda^{*}, \mathrm{M}^{*}$ refer to literature [7] or [8]. The influence of suction automatically brings in the equation [9]. After the above theory determined the parameters, we calculate $\mathrm{p}^{\prime} \sim \varepsilon_{v}^{p}$ and $\mathrm{q} \sim \varepsilon$ curve (or the axial strain curve) according to the test path. Then compare it with the test results. If not consistent, it can be used in engineering after adjustment to conform or roughly consistent. Stress state of triaxial test is axisymmetric. When Small and medium principal stress ratio increase, the dilatancy of soil and $M *$ increase. There is a problem of smaller than true when $M *$ measured by triaxial test is using in non axisymmetric stress state.

\section{CALCULATION EXAMPLE}

Figure 1 is axial compression curve measured values and theoretical values of remoulded soil. Plastic volumetric strain and shear strain of axis compression were used formula (9), (10) respectively.The calculation formula of elastic volumetric strain and axial strain, respectively

$$
\varepsilon_{v}^{e}=\frac{k}{1+e_{0}} \ln \frac{p^{\prime}}{p_{0}^{\prime}}
$$

$$
\varepsilon_{1}=\varepsilon+\frac{\varepsilon_{V}^{p}+\varepsilon_{v}^{e}}{3}
$$

The physical properties of the soil in text refer to literature [10], calculation parameters are shown in table 1. The calculation results show that the shear shrinkage, dilatancy corresponding epsilon $\varepsilon_{1}$ calculated value corrected with 0.4 and 0.17 respectively. The calculated value of $\varepsilon_{\mathrm{v} 0}^{\mathrm{p}}$ needn't fix while shear shrinkage, dilatancy corrected by 0.17 . 


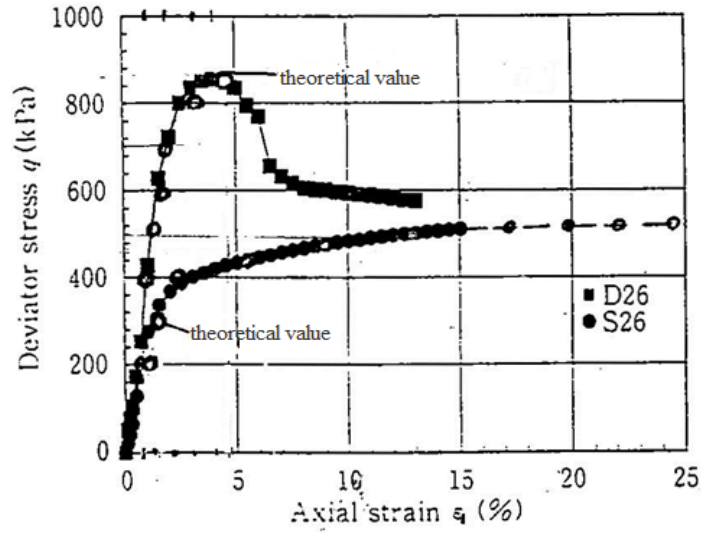

(a) Saturation $\left(\sigma_{3=} 500 \mathrm{kPa}\right)$

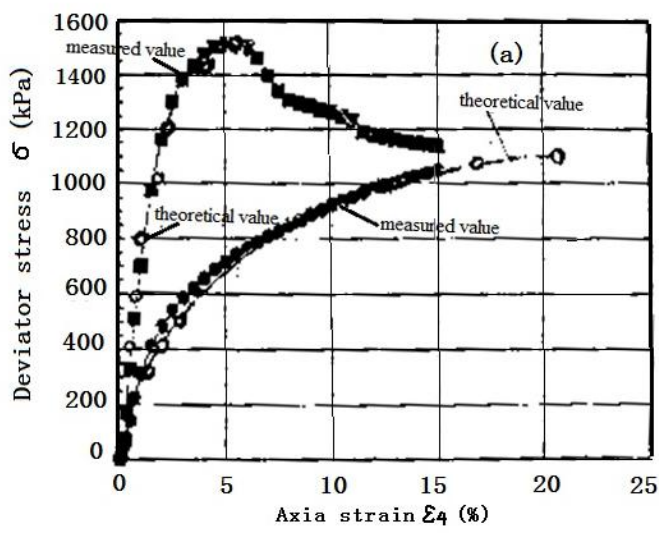

(b) Unsaturated $\left(\sigma_{3}=200 \mathrm{kPa}\right.$, suction $\mathrm{s}=60 \mathrm{kPa}$, saturation $\left.\mathrm{Sr}=40 \%\right)$

Figure 1. Measured values compared with the theoretical value of $\varepsilon_{1} \sim q$ curve

TABLE I. CALCULATION PARAMETER LIST

\begin{tabular}{|c|c|c|c|c|c|c|c|c|}
\hline category & $\mathrm{e} 0$ & M & $\mathrm{M}^{*}$ & $\lambda^{*}$ & $\kappa$ & $\begin{array}{l}\mathrm{P}_{\mathrm{m}} \\
\mathrm{kPa}\end{array}$ & $\mathrm{p}_{\mathrm{b}}$ & note \\
\hline $\begin{array}{c}\text { Saturated } \\
\text { hardening }\end{array}$ & 1.00 & 1.30 & 1.30 & 0.10 & 0.02 & - & & \multirow{4}{*}{$\begin{array}{c}\text { Soil particle density } \rho s=2.65 \text {, bound water } \\
\text { saturation Sro } \approx 10 \% \text {, unsaturated soil. } M \text { and } \\
\mathrm{M}^{*} \text {, have joined the pm influence, its value } \\
\text { is } 0.1\end{array}$} \\
\hline Saturated soft & 0.74 & 1.10 & 1.60 & 0.10 & 0.02 & - & & \\
\hline $\begin{array}{l}\text { Unsaturated } \\
\text { hardening }\end{array}$ & 1.00 & $1.30+0.1$ & 1.40 & 0.08 & 0.02 & 20 & 0 & \\
\hline $\begin{array}{l}\text { Unsaturated } \\
\text { softening }\end{array}$ & 0.74 & $1.1+0.1$ & 1.86 & 0.08 & 0.02 & 20 & 0 & \\
\hline
\end{tabular}

\section{CONCLUSION AND THE PROBlem to CONTINUE to STUDY}

A. The increase of geotechnical yield stress is due to the plastic volume strain, Plastic potential defined by plastic volume work, taking regular octahedron for the study, fixed loading direction, the assumption of the traditional plastic potential theory is reasonable for the shear shrinkage soil, volume dilatancy of soil state began to yield stress that is doing negative work, formula of ilyushin do not apply

B. Plastic volumetric strain dilatancy cannot produce in terms of isotropic pressure, Shear dilatancy by decomposition of the energy equation for the shrink, yield function comes with plastic volume expansion line, the former is the same as yield function of Cambridge model, shearing shrink age, expansion line is to zero automatically.

C. Provisions of the vertical load, actual change in the direction of loading, to increase the lateral pressure to change the stress state firstly, and then increase the vertical pressure. The correlation between stress increment and plastic strain increment are achieved by the two loading. It can be used to calculate the deformation of the foundation by using the method of layered synthesis.

D. Parameters are determined by the triaxial test. When saturated soil shear shrinkage is three, and the dilatancy is four; when it is used to normally consolidated soil, a water curve is added. The relationship between the strength parameter and the internal stress is added, when it is used to the super consolidation of soil, all measurement on unsaturated triaxial test.

E. The results of the application showed that the calculation result of the shear strain is too large; energy equation need to be modified, the method is very simple, with a reduction factor which can achieve satisfactory effect.

The deformation and failure characteristics of the materials need to be measured through experiment. This paper put the elastic-plastic theory simplified as: using the energy conservation principle resolution the stress-strain relationship of the test, according with mechanics principle, the test defects are classified into the correction and error categories.can simplify theory, convenient for engineering application. As a new method of research, there are a lot of content, such as fixed law of energy equation, stress path folding, spindle rotation, cyclic loading, the special soil, rock and so on, which need to be expanded in the future and indepth study.

\section{REFERENCES}

[1] Xueyan Zhang, Peng wang Yan. 2004. Basis of geotechnical plastic mechanics. Tianjin: Tianjin university press $[\mathrm{M}]$. 
[2] Yingren Zheng, Liang Kong. 2006. Geotechnical plastic mechanics. Beijing: China building industry press $[\mathrm{M}]$.

[3] Zongze Yin. 2007. Earthwork principle [M]. Beijing: China water conservancy and hydropower press.

[4] Meifeng Cai. 2002. Rock mechanics and engineering. Beijing: science press $[\mathrm{M}]$.

[5] Zhiling Wang. 2014.9. Saturated soil shear strength theory and application research, The fifth China water conservancy and hydropower engineering rock and soil mechanics and engineering academic symposium on $[\mathrm{M}]$. Beijing: water conservancy electric power press.

[6] Daito Karube, Sheji Kato, Hamada, K.and Hond, M. 1996. The ralationship between the mechanical behabior and the state of porewater in unsaturated soil [J]. Proceedings of civil society, (535):83-92.
[7] Zhiling Wang, Minzhi Li \& Xu Wang. 2008. Effective stress principle of unsaturated soil research and compression index [J] Journal of hohai university (natural science edition), 36 (supplement), The effective stress principle of unsaturated soil.

[8] Daito Karube, Hoda, M, Sheji kato. 1997. The relationship between the shearing characteristics and the composition of porewater in unsaturated soil [J]. Proceedings of the civil society, (575):49-58.

[9] Yuji KOHGO and HidekiMORIYAMA. 1997. Volume change and shear behavior of unsaturated silt and sand/clay mixed soil under triaxial stress conditions [J]. Proceedings of civil society of agriculture, 65(6):47-5.

[10] Yuji KOHGO and HidekiMORIYAMA. 1998. Interpretation of the Mechanical Behavior of Unsaturated Soils under Triaxial Stress Conditions based on an Elastoplastic Model [J], proceedings of civil society of agriculture (2): 35-49. 